\title{
Single Qutrit Entanglement
}

\author{
Sinem Binicioglu, Alexander A. Klyachko, Alexander S. Shumovsky \\ Bilkent University 06800 Ankara, Turkey \\ sinem@fen.bilkent.edu.tr
}

\begin{abstract}
We discussed a recent approach to quantum entanglement. The approach is based on presetting of basic observables of quantum system. Entangled states are interpreted as states with maximal amount of uncertainty of all basic observables.

(C) 2007 Optical Society of America

OCIS codes: 030.1640 Coherence ; 270.6570 Squeezed
\end{abstract}

\section{Introduction}

Entanglement is usually associated with quantum nonlocality or violation of classical realism [1], [2], [3]. Physically this is caused by the quantum correlations between the parts of the system. Once created, those correlations keep on existing even after the spatial separation of parts. This notion does not contain any quantification of distance between separated entangled parts of a quantum system. Thus, it seems to be natural to assume that quantum system with strongly correlated intrinsic parts may manifest entanglement independent of distance between the parts and hence even as a local object without spatial separation of parts [4], [5], [6], [7], [8].

The quantum nonlocality can be expressed in terms of violation of different Bell-type conditions of classical realism. However, unentangled states can also manifest violation of those conditions [9], [10], [11]. As a matter of fact, violation of Bell-type conditions indicates the absence of hidden classical variables in quantum mechanics rather than entanglement. This allows us to conclude that nonlocality and violation of classical realism alone are not the essential sign of entanglement and that there is no physical prohibition for the existence of entanglement of local objects (particles) caused by quantum correlations of their intrinsic degrees of freedom [4], [5], [6], [7], [8].

Another common opinion is that the entanglement of multipartite systems defined in the Hilbert space $\mathcal{H}=$ $\mathcal{H}_{A} \otimes \mathcal{H}_{B} \otimes \cdots$ can be associated with the nonseparability of states $\psi \in \mathcal{H}$ with respect to the parts of the system. This statement, which is undoubtedly valid in the case of bipartite systems, does not have a lucid sense for multipartite entanglement. Three-qubit states, whose classification has been constructed in Ref. [12] can be considered as an example.

Thus, it seems reasonable to set aside the criteria of entanglement based on nonlocality, violation of classical realism, and nonseparability, and to focus attention on physical manifestations of entanglement in the process of measurement of quantum observables.

\section{Entanglement and Quantum Fluctuations}

The natural question is now, how many physical observables should be measured in order to conclude that a given state of a certain system is entangled [13]? This question has extremely high importance for understanding of physical essence of entanglement and its quantification. Besides that, this question has a quite practical meaning in connection with test of sources of entangled states [14].

In a recent approach (for recent review, see Ref. [8]), it has been proposed to begin the analysis of entanglement with the choice of independent basic observables that can be associated with the orthogonal basis of a certain Lie algebra $\mathcal{L}$. The corresponding Lie group $G=\exp (i \mathcal{L})$ defines the dynamic symmetry of the physical system under consideration.

Within the approach of Refs. [9], [4], [15], the complete entanglement is interpreted as manifestation of quantum uncertainties of all basic observables at their extreme. By complete entanglement we mean here the maximal entanglement that can be achieved by pure states. Complete entangled states $\psi_{C E}$ can be defined in terms of maximum of total variance. This definition has a simple physical meaning. It associates complete entanglement with the maximal amount of quantum uncertainty in a given system. Validity of this definition in some known cases of completely entangled states of multipartite systems has been shown in a number of papers (see Ref. [8] for references).

Qutrit is usually associated with ternary unit of quantum information. Instructiveness of this example consists in the relativity of entanglement with respect to the choice of dynamic symmetry G of ternary quantum physical system. Namely, one can choose either $\mathrm{G}=\mathrm{SU}(3)$, which implies eight basic observables (Gell-Mann matrices) [16] or 


\section{CMI50.pdf}

$\mathrm{G}^{\prime}=\mathrm{SU}(2)$, of the physical system, which requires only three basic observables (spin-1 operators). Just the latter case of a single spin-1 system may manifest entanglement without division of the system into separated parts [4], [6], [7]. We call this case the spin-qutrit system.

\section{Entanglement in a single spin-qutrit system}

Single-particle entanglement is caused by quantum correlations between intrinsic degrees of freedom of the particle. There is a transparent formal correspondence between symmetric states of two qubits and single $\mathrm{SU}(2)$ qutrit, coming from the Clebsch-Gordon decomposition $\mathcal{H}_{2} \otimes \mathcal{H}_{2}=\mathcal{H}_{3} \oplus \mathcal{H}_{A}$. If we assume that the singlet state is forbidden because of some physical reasons, then the system of two qubits becomes exactly equivalent to the spin-qutrit system. An example of some considerable interest is provided by the so-called biphoton.

$\left|\psi_{0}\right\rangle=|0\rangle$ and $\left|\psi_{ \pm}\right\rangle=\frac{1}{\sqrt{2}}(|1\rangle \pm|-1\rangle)$ states give the maximum of total variance, they are completely entangled qutrit states of a single spin-qutrit system (respectively $\left.\left.\frac{1}{\sqrt{2}}(|\uparrow \downarrow\rangle+\downarrow \uparrow\rangle\right), \frac{1}{\sqrt{2}}(|\uparrow \uparrow\rangle \pm \downarrow \downarrow\rangle\right)$ states of two-qubit system).

It is known that an entangled two-qubit state is associated with the $\mathrm{SU}(2)$ squeezed states, while unentangled states are the SU(2) coherent states [9], [10]. This interpretation is valid for the entangled and unentangled states of a single spin-qutrit as well.

$\mathrm{SU}(2)$ coherent states for a spin $s$, are defined as $|\alpha\rangle=D(\alpha)|-s\rangle, \quad \alpha \in C$. The displacement operator amounts to an $\mathrm{SU}(2)$ rotation. This means that every spin coherent state is just a state with minimal spin projection $-s$ onto some direction, which can be chosen as a new quantization axis. Thus, there is no principle difference between the spin coherent state and state $|-s\rangle$. Thus, spin coherent state is as unentangled as the state $|-s\rangle$.

Following Kitagawa and Ueda [17], we call spin state $\xi$ to be squeezed iff $V_{r}(\xi)<s / 2$ for some direction $r \perp \vec{s}$, where $\vec{s}$ is the direction of the average spin vector. This means that in a coordinate system with the $z$-axis along the average spin vector $\vec{s}$, we always have $V_{x}(\xi)+V_{y}(\xi) \geq s$ in contrast to the spin-coherent state. It is easy to check that this condition of squeezing is valid for the states $\left|\psi_{0}\right\rangle$ and $\left|\psi_{ \pm}\right\rangle$, therefore they are squeezed.

Some spin-squeezed states (for spin- $s \geq 1$ ) can be constructed by means of the squeezing operator [17] $\mathcal{S}(\xi)=\exp \left[\left(\xi^{*} S_{-}^{2}-\xi S_{+}^{2}\right) / 2\right]$. Here $\varphi=\arg \xi$. At $\xi=\pi / 4+k \pi$, this state coincides with $\left|\psi_{ \pm}\right\rangle$and hence manifests complete entanglement. Note that in the case of a single qubit, the squeezing operator is simply the identity operator. For the single spin-qutrit system, coherent states are unentangled while squeezed states manifest entanglement like in the case of conventional two-qubit states.

We showed that the single-particle entanglement has all important properties of conventional two-qubit entanglement, its unentangled states are the spin-coherent states like those of two qubits [9], [10] In turn, the single-particle entangled states are squeezed like the two-qubit entangled states. Note that association of unentangled and coherent states on the one hand and entangled and squeezed states on the other hand blends well with the concept of entanglement as manifestation of quantum uncertainties at their extreme.

Besides biphoton, we considered number of physical examples for single spin qutrit entanglement. We also showed violation of Bell type inequalities by a single particle. For further and detailed discussion and references therein see [18].

\section{References}

1. A. Einstein, B. Podolsky, and N. Rosen, Phys. Rev. 47, 777 (1935).

2. J.S. Bell, Rev. Mod. Phys. 38, 447 (1966).

3. M.A. Nielsen and I.L. Chuang, Quantum Computations and Quantum Information (Cambridge University Press, New York, 2000).

4. A.A. Klyachko and A.S. Shumovsky, J. Opt. B: Quant. and Semiclas. Optics 5, S322 (2003).

5. S.J. van Enk, Phys. Rev. A 67, 022303 (2003); Phys. Rev. A 72, 064306 (2005).

6. H. Barnum, E. Knill, G. Ortiz, R. Somma, and L. Viola, Phys. Rev. Lett. 92, 107902 (2004).

7. M.A. Can, A.A. Klyachko, and A.S. Shumovsky, J. Opt. B: Quant. Semiclass. Opt. 7, L1 (2005).

8. A.A. Klyachko and A.S. Shumovsky, J. Phys: Conf. Series 36, 87 (2006); E-print quant-ph/0512213.

9. A.A. Klyachko, E-print quant-ph/0206012.

10. H. Barnum, E. Knill, G. Ortiz, and L. Viola, Phys. Rev. A 68032308 (2003).

11. A.A. Klyachko, J. Phys.: Conf. Series 36, 87 (2006).

12. A. Miyake, Phys. Rev. A 67, 012108 (2003).

13. J.M.G. Sancho, S.F. Huelga, Phys. Rev. A 61, 042303 (2000).

14. M. Hayashi, B.-S. Shi, A. Tomita, K. Matsumoto, Y. Tsuda, and Y.-K. Jiang, E-print quant-ph/0603254.

15. A.A. Klyachko and A.S. Shumovsky, J. Opt. B: Quantum and Semiclass. Opt. 6, S29 (2004).

16. C.M. Caves and G.J. Milburn, Opt. Commun. 179, 439 (2000).

17. M. Kitagawa and M. Ueda, Phys. Rev. A 47, 5138 (1993).

18. S. Binicioglu, M. A. Can, A. A. Klyachko, A. S. Shumovsky, E-print quant-ph/0604182. 